\title{
BIBLIOGRAFÍA
}

- David Harvey. Breve historia del neoliberalismo.

- JUAN PABLO MATEO TOMÉ. Crisis, política monetaria y tipos de interés: fuente del neoliberalismo y la financiación.

- Joseph E. Stiglitz. ¿El fin del neoliberalismo?

\section{DINÁMICA MOLECULAR COMO TÉCNICA DE SIMULACIÓN}

\author{
Lorena Paola Cárdenas Espinosa \\ Grupo de Física Teórica y Computacional
}

\section{Resumen}

Para simular sistemas físicos compuestos por muchas partículas existen diversos métodos matemáticos, cada uno con ciertas ventajas y desventajas, siendo unos más eficientes que otros; cuando exigimos que el método sea de bajo requerimiento computacional sin que deje de ser eficiente, aparece la Dinámica Molecular como un método capaz de reproducir de la mejor manera cualquier modelo físico. En este trabajo se hace una breve descripción de éste método.

\section{INTRODUCCIÓN}

La Dinámica Molecular es un método computacional muy sencillo y eficiente, capaz de reproducir de manera muy aproximada, el comportamiento de un sistema físico donde participen gran cantidad de partículas. Puede utilizarse cualquier lenguaje de programación y un computador sin características especiales. Sin embargo, para tener mejores resultados se requiere la introducción de muchas más partículas, siendo necesario un computador con mucha más velocidad que capacidad de almacenamiento. Para aplicaciones mucho más sofisticadas se utiliza procesamiento en paralelo en computadores ultrarápidos.

\section{DİNÁMICA MOLECULAR}

La Dinámica Molecular (DM) (en ingles Molecular Dynamics) es una técnica de cómputo que permite describir la evolución en el tiempo de un sistema físico partiendo de las causas que hacen que el sistema cambie sus propiedades a lo largo del tiempo.

En general, los sistemas moleculares son complejos y consisten de un gran 
número de partículas, por lo cual sería imposible encontrar sus propiedades de forma analítica. Para evitar este problema, la DM utiliza métodos numéricos. Representa un punto intermedio entre los experimentos y la teorfa. Puede ser entendida como un experimento en la computadora.

En DM las partículas interactúan unas con otras mediante potenciales. Estos potenciales se manifiestan como fuerzas que actúan de manera instantánea entre las partículas haciendo que éstas cambien sus posiciones. En el momento en que estas posiciones relativas varían, se produce también un cambio en las fuerzas que actúan sobre cada partícula, haciendo nuevamente que las posiciones varién nuevamente, lo cual se convierte en un proceso cíclico de interacción y movimiento [1].

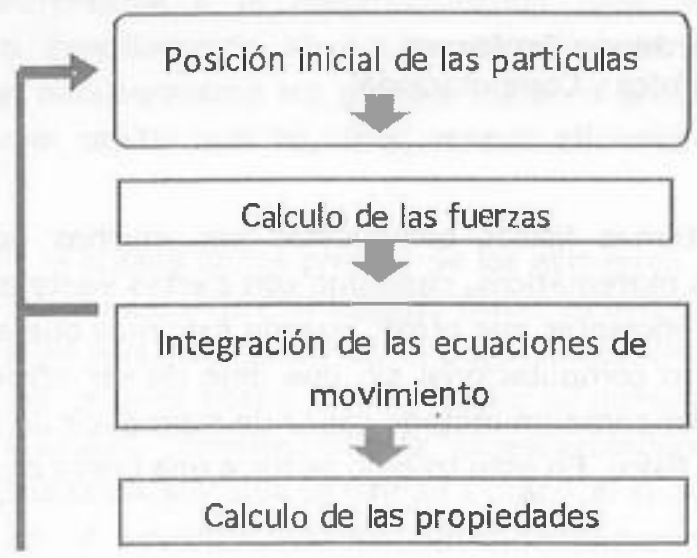

Fig1: Diagrama de simulación de DM

Esta técnica trabaja desde una aproximación de la física clásica de un sistema de partículas que interactúan entre sí, por to cual en DM las partículas se mueven de acuerdo a las leyes de la mecánica Clásica, más precisamente de acuerdo a la segunda Ley del Movimiento de Newton:

$$
F_{i}=m_{i} a_{i}
$$

En la ecuación 1 se expresa la fuerza $F_{1}$ sobre cada partícula $i$ en un sistema constituido por $N$ partículas, siendo $m$, la masa de cada una de las partículas $y a_{1}$ la aceleración de cada partícula dada por la ecuación 2 , donde $r_{1}$ es la posición para cada una de las partículas.

$$
a_{i}=\frac{d^{2} r_{i}}{d t^{2}}
$$

La DM se considera como una técnica computacional determinista, ya que teniendo las condiciones iniciales del problema en este caso, posiciones y 
velocidades, se puede determinar completamente su evolución temporal.

El resultado de una simulación de dinámica molecular son las posiciones $X$ y velocidades $\mathrm{V}$ de cada átomo de la molécula, para cada instante en el tiempo discretisado. A esto se le llama trayectoria. [2]

\section{PRINCIPIOS FÍSICOS}

El ingrediente principal para la simulación es un sistema físico de partículas que interactúan por medio de fuerzas o físicamente un conjunto micro canónico, en este conjunto, el sistema esta aislado, su volumen no se altera, no hay intercambio de masa ni de energía con el entorno.

Luego se tiene un sistema de $\mathrm{N}$ partículas con coordenadas y velocidades definidas inicialmente, con las cuales se puede plantear la ecuación 3, donde se tiene la fuerza en función del gradiente de la función potencial la cual depende de las posiciones relativas entre las partículas.

$$
F_{i}=-\nabla_{x_{\mathrm{i}}} \mathrm{V}\left(\mathrm{r}_{1}, \mathrm{r}_{2}, \ldots, \mathrm{r}_{\mathrm{N}}\right)
$$

El potencial se puede describir por la interacción entre pares de partículas, para $\mathrm{N}$ partículas este potencial se obtiene por la sumatoria de las interacción entre las $\mathrm{N}$ partículas, en la ecuación 4 , se tiene que $\varphi\left(\left|r_{i}-r_{j}\right|\right)$ es una función de interacción entre pares de partículas. Se realizan dos sumatorias donde j > i lo cual implica que la contribución debido a la interacción de cada par de partículas sea contada una sola vez.

$$
V\left(\mathrm{r}_{1}, \mathrm{r}_{2}, \ldots, \mathrm{r}_{\mathrm{N}}\right)=\sum_{i, j \rightarrow i} \varphi\left(\left|r_{i}-r_{j}\right|\right)
$$

$V$ Es la función de energía potencial que describe las atracciones y repulsiones que sienten los átomos entre sí debido a las fuerzas intermoleculares de las moléculas.

\section{ALGORITMO USADO EN DINÁMICA MOLECULAR}

En la simulación de DM las partículas son representadas como masas puntuales, que interactúan a través de potenciales los cuales dependen de la distancia de separación, entonces la fuerza $F_{1}$ que actúan sobre cada partícula se puede obtener por medio de la ecuación 5

$$
F_{i}\left(r_{i}\right)=\sum_{i, j>i} F_{i, j}\left(r_{i}\right)=-\sum_{i, j>i} \nabla V_{i, j}\left(r_{i}\right)
$$

Los primeros trabajos sobre DM con potenciales continuos se hicieron en 1964 (simulation of argon by Rahman) [3]. Por medio del método de diferencias finitas: la integración se fracciona en varios términos pequeños, cada uno separado en el tiempo por un incremento de tiempo fijo dt.

El motor de la simulación de DM es el algoritmo de integración de Verlet [4]. Fue 
desarrollado por Loup Verlet, la idea básica parte de expandir en serie de Taylor dos expresiones para la posición dependiente del tiempo, una para un paso adelante, ecuación 6, y la otra un paso atrás, ecuación 7.

$$
r(t-\Delta t)=r(t)-v(t) \Delta t+\frac{1}{2} a(t) \Delta t^{2}-\frac{1}{6} b(t) \Delta t^{3}+o\left(\Delta t^{4}\right)
$$

Donde $r(t)$,son las posiciones, $v$ la velocidad, a la aceleración, y b la tercera derivada de $r$ con respecto al tiempo. Sumando las dos expresiones y reordenando se obtiene la ecuación 8 , observando un error de truncamiento del algoritmo cuando el sistema avanza con un paso de tiempo $\Delta t$ es del orden de:

$$
v(t)=\frac{r(t+\Delta t)-r(t-\Delta t)}{2 \Delta t}
$$

\section{CONCLUSIONES}

-Esta técnica presenta un compromiso entre costo computacional y fiabilidad en los resultados, ya que se utilizan las Ecuaciones de Newton, que son más sencillas que las de la mecánica cuántica. Es por ello que muchas propiedades que pueden resultar de interés, como por ejemplo la formación o ruptura de enlaces no puedan ser estudiadas mediante este método ya que no contempla estados excitados o reactividad.

-La Dinámica Molecular es un método determinístico, con lo que queremos decir que el estado del sistema en un tiempo dado en el futuro se puede predecir a partir del estado actual.

-Los métodos de integración son aproximaciones, por lo cual se pueden presentar errores asociados a estos, tales como errores de truncamiento o errores de redondeo, pero estos errores se pueden controlar variando el valor de paso del tiempo.

\section{BIBLIOGRAFÍA}

- TORRES, Edmanuel. Como Simular un gas de Argón por la técnica de Dinámica Molecular. Versión 0.5. 19 de abril de 2008.

- B. J. Alder y T. E.Wainwright, Studies in Molecular Dynamics. I. General Method, The Journal of Chemical Physics.

- A. Rahman, Correlations in the Motion of Atoms in Liquid Argon, Physical Review136, (1964).

-Loup Verlet, Computer "Experiments" on Classica! Fluids. I. Thermodynamical Properties of Lennard-Jones Molecules, Physical Review 156, (1967), 98. 